\title{
P01-1-8
}

\section{Adolescent mouse hippocampal function was impaired by prenatal nicotine exposure}

\section{Takayoshi Mamiya $^{1,2}$, Shota Tanase ${ }^{1}$, Maki Hada ${ }^{1}$, Shunsuke Kato ${ }^{1}$, Shino Takeuchi ${ }^{1}$, Daisuke lbi ${ }^{1}$, Toshitaka Nabeshima ${ }^{1,2,3}$, Masayuki Hiramatsu ${ }^{1,2}$}

${ }^{I}$ Faculty of Pharmacy, Meijo University, Japan, ${ }^{2}$ NPO Japanese Drug Organization of Appropriate Use and Research, Japan, ${ }^{3}$ Graduate School of Health Sciences, Fujita Health University, Japan

It is not clear how prenatal nicotine exposure (PNE) may cause cognitive impairment in offspring. In this study, we investigated whether the exposure to nicotine $(0.2 \mathrm{mg} / \mathrm{mL}$ in drinking water with $2 \%$ sucrose $)$ during E14-P0 impaired hippocampus-dependent learning and memory in adolescence. In the hippocampal CA1 region, the induction and maintenance of $\mathrm{N}$-methyl-D-aspartate (NMDA) receptor dependent long-term potentiation (LTP) was diminished by PNE, whereas the paired-pulse facilitation was not affected. Behaviorally, PNE impaired contextual- but not tonedependent fear memory in 7- to 8-week-old mice. Both impairments were attenuated by the repeated co-treatment with methyllycaconitine $(5 \mathrm{mg} / \mathrm{kg}$ s.c.), a nicotinic alpha 7 receptor antagonist. The results suggest that the nicotinic alpha 7 receptor dependent plasticity during embryonic period may be required for NMDA receptor-related long-term memory formation, and that PNE may disrupt this form of plasticity. 